\title{
Critical Appraisal of Published Literature in Specialty General Surgical Examinations in the United Kingdom
}

\author{
Badri Man Shrestha ${ }^{1}$ \\ 'Sheffield Kidney Institute, Sheffield Teaching Hospitals NHS Trust, Sheffield, S5 7AU, UK.
}

\section{ABSTRACT}

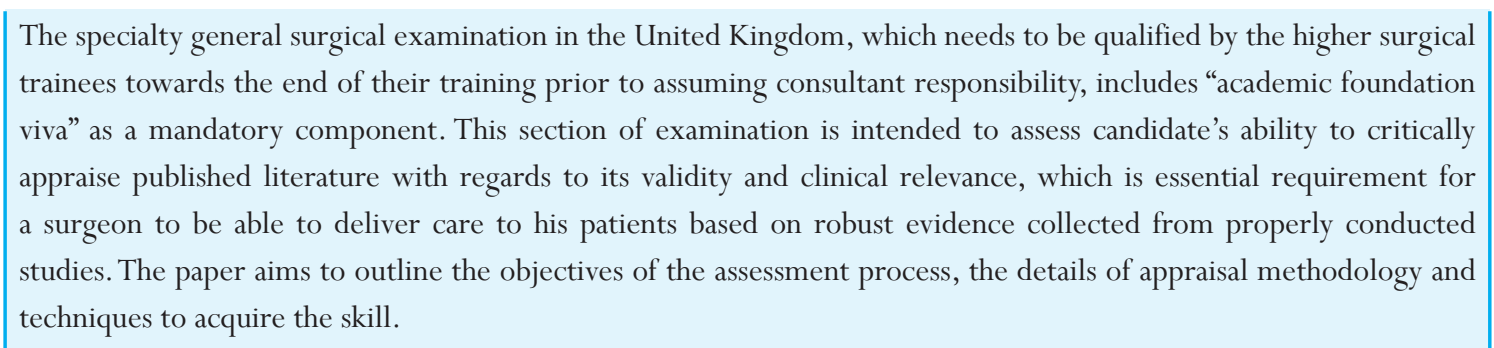

\section{INTRODUCTION}

Clinicians, in the current era, involved in managing patients in all specialties must be accustomed to read published papers relevant to their clinical practice on a regular basis, be trained to critically evaluate their authenticity and clinical relevance and be prepared to change their practice for achieving best outcomes. ${ }^{1}$ This paper aims to outline the format and objectives of the academic foundation viva of the specialty general surgical examinations in the United Kingdom where critical appraisal of published literature is a mandatory component.

\section{GENERAL SURGICAL TRAINING}

In the United Kingdom, general surgical specialty training consists of foundation years (2 years), core surgical training (2 years), specialist registrar (6 years), fellowship (6 months - 1 year; optional) and research (2-3 years leading to MD / PhD: optional) prior to being eligible to work independently as a consultant. The subspecialties of general surgery include upper gastrointestinal, hepatobiliary-pancreatic, colorectal, breast, endocrine, vascular and transplantation surgery. At the final 2 years of training, candidate must pass the Intercollegiate Specialty Board Examination in their respective specialty leading to award of the diploma of Fellowship \{FRCS (Gen)\}. ${ }^{2}$ One of the components of examination consists of academic viva where candidates are given a published paper related to their specialty to appraise within 30 minutes, which is followed by assessment by two examiners within 15 minutes. The lead examiner assessing the candidate has a different specialty interest and the second examiner who belongs to the same specialty as the candidate, participates in the assessment and marking. The purpose of examination by an examiner of different specialty is to assess the candidate's ability to critically appraise the paper rather than assess the depth of knowledge on the subject. However, the second examiner belonging to the same specialty can complement the lead examiner, if needed, with relevant advancements in his specialty. ${ }^{3}$

\section{APPRAISAL OF THE PAPER}

Candidates are expected not just to present the summary of the paper, but to consider whether the study undertaken is of clinical relevance. The ability of the candidate to critically evaluate of each section of the paper is important. Publication of a manuscript in a reputable journal does not necessarily indicate that the study has been conducted properly or is of good quality. It is important to appreciate that the peer-reviewers play a very important role in the decision-making process on any manuscript, hence their expertise and experience are reflected on the quality of the paper. The papers given for appraisal include randomised -controlled trials, meta-analysis and cohort studies, which do often have both strengths and limitations.

While evaluating a paper, it is important to consider 
specific points in each section. The title and abstract should accurately reflect the content of the paper. It is relevant to bear in mind the institutional affiliation of authors and the impact factor of the journal. The introduction section should include the background, aim of the study and proposed hypothesis that is being tested. The methodology section should clearly define the selection criteria of patients including the inclusion and exclusion criteria to eliminate selection bias, power calculation, end points and statistical methods. The results section should clearly depict the finding without involvement of any type I and II errors. The follow-up should be adequate and data analysis should be done on an intention to treat basis. The discussion section should provide thorough discussion of the results comparing with the existing published literature. Any novel observations should be highlighted. All conclusions made should be supported by the data presented and clinical relevance of the data must be discussed. Strength and limitations of the study needs to be highlighted. Citation of the references should be adequate including majority of them should be published within previous 10 years. Any conflict of interest must be declared by individual authors. ${ }^{4}$

\section{ACQUISITION OF SKILL}

To be able to satisfy the objective of the examination, the candidate should have been in practice of regular reading and appraisal of published papers in contemporary professional journals. Discussion of papers in departmental meetings, "journal club", on a regular basis help develop the skill. In postgraduate training programmes, although journal clubs involving review of published papers are routinely done, having academic viva as one of the mandatory components of examination, makes the training more effective.

The pragmatic approach to remain in touch with journals relevant to one's clinical practice is by setting up "email alerts", which are free of charges, so that the publications are reviewed as soon as they are published. Relevant articles should be read in detail, downloaded and stored in a folder for future usage. Besides specialty journals, several landmark research works are published in generic journals such as the New England Journal of Medicine, The Lancet and British Medical Journal, which are published on weekly basis should be read regularly.

This paper has highlighted the current curriculum of examination, including academic viva, in the specialty general surgical examination in the United Kingdom and laid emphasis on the need for inclusion of critical appraisal of literature in the post-graduate surgical training curriculum through regular interactive sessions followed by their formal assessment at the exit examination settings.

\section{REFERENCES}

1. Tseng TY, Dahm P, Poolman RW, Preminger GM, Canales BJ, Montori VM. How to use a systematic literature review and meta-analysis. J Urol. 2008;180(4):1249-56.[Science Direct]

2. JCST — [Internet]. [cited 2017 Sep 2]. Available from: http:/ / www.jcst.org/key-documents/docs/a-referenceguide-for-postgraduate-specialty-training-in-the-uk-goldguide-2016

3. General Surgery [Internet]. [cited 2017 Sep 2]. [Link]

4. Sena ES, Currie GL, McCann SK, Macleod MR, Howells DW. Systematic reviews and meta-analysis of preclinical studies: why perform them and how to appraise them critically. J Cereb Blood Flow Metab. 2014;34(5):737-42. [Full Text]

5. Tseng TY, Dahm P, Poolman RW, Preminger GM, Canales BJ, Montori VM. How to use a systematic literature review and meta-analysis. J Urol. 2008; 180(4): 1249-56. [Science Direct] 